\title{
The Auxiliary Effect of Different Bacterial Groups on the Degradation of Organic Matter in Refinery Wastewater by Bacillus Licheniformis
}

\author{
Yong Chen ${ }^{1,}$, Yue Jiao ${ }^{2}$,XiaoLi Wen ${ }^{3}$ \\ ${ }^{1}$ School of environment and BioengineeringHenan University of Engineering, Zhengzhou, Henan, China \\ ${ }^{2}$ Gongyi branch of Zhengzhou Ecological Environment Bureau,Zhengzhou, Henan, China \\ ${ }^{3}$ School of Nursing, Henan Technical Institute,Zhengzhou, Henan, China \\ *Corresponding Author.
}

\begin{abstract}
In this paper, the biodegradation of organic compounds in refinery wastewater was studied. Different microbial communities suitable for the treatment of refinery wastewater were selected to degrade the pretreated wastewater. The results showed that the degradation efficiency of COD and ammonia nitrogen was 59.4\% and 56.7\% respectively; the degradation efficiency of COD and ammonia nitrogen was $50.2 \%$ and $60.6 \%$ respectively. It can be used as the dominant bacteria for further domestication and cultivation of petrochemical wastewater biological treatment.
\end{abstract}

Keywords: Refinery wastewater,biological treatment, bacillus licheniformis, degradation effect

\section{Introduction}

Petrochemical wastewater is produced in the process of petrochemical products processing. The quality and quantity of wastewater fluctuate greatly, the composition is complex, the concentration of organic matter is high, and most of them are difficult to degrade. Most of the secondary treatment of petrochemical wastewater is biochemical treatment process, which has the problem of insufficient activity of activated sludge in biochemical reaction tank. The main reason is that the microbial population of biochemical reaction pool has not strong ability to degrade COD and ammonia nitrogen in wastewater[1]. Therefore, finding one or several microorganisms that can efficiently degrade CODCR and ammonia nitrogen in petrochemical wastewater can effectively improve the treatment effect of petrochemical wastewater[2,3].

This study is mainly based on Bacillus, assisted by different microbial groups, analyzes the treatment effect, finds out the bacterial groups with significant purification effect, studies the growth law of degrading microorganisms, and provides a certain technical basis for the biological treatment of petrochemical wastewater.

\section{Experimental Subject}

2.1Wastewater quality

The wastewater samples used in this study are all taken from the second wastewater treatment plant of a refinery wastewater treatment plant[4]. The water intake point is located at the entrance of the biochemical reaction tank of the wastewater treatment plant. The pretreatment processes mainly include grid, regulating tank[5], oil separator and pressurized dissolved air flotation. The influent water quality of wastewater is shown in Table 1. 
Table 1 Wastewater quality

\begin{tabular}{|c|c|c|c|}
\hline $\mathrm{COD}_{\mathrm{Cr}}$ & $\mathrm{NH}_{4}-\mathrm{N}$ & $\mathrm{TP}$ & $\mathrm{pH}$ \\
\hline $606.1 \mathrm{mg} / \mathrm{L}$ & $65.8 \mathrm{mg} / \mathrm{L}$ & $\mathrm{ND}$ & 6.82 \\
\hline
\end{tabular}

\subsection{Bacterial agents}

In this experiment, the solid bacterial agent is used. In addition to microorganisms, the bacterial agent also contains a large number of nutrients[6]. Direct inoculation and sewage will cause the change of nutrients in sewage. Therefore, in this experiment, the solid bacterial agent is transferred into bacterial solution, and then the bacteria are separated for experimental research.

According to the relevant literature, five microbial combinations were selected, which were: (1) Bacillus licheniformis(2) Bacillus licheniformis+yeast(3) Bacillus licheniformis+yeast+Micrococcus(4) Bacillus licheniformis+Pediococcus(5) Bacillus licheniformis + Enterococcus[7].

\section{Scereening of Flora}

Take1Lwastewatersample, add $1 \mathrm{~g}$ glucose and $0.065 \mathrm{~g}$ sodium dihydrogen phosphate, stir and dissolve, adjust $\mathrm{pH}$ to 7.5 , and sub pack into $250 \mathrm{ml}$ conical flask, each bottle is $100 \mathrm{ml} .0 .1 \mathrm{ml}$ of the prepared bacterial solution was aspirated with a pipette gun in the ultra clean table, added into a $10 \mathrm{ml}$ centrifuge tube, and then $5 \mathrm{ml}$ sterile water was added to the centrifuge tube to shake up. After centrifugation for 25 minutes at $5000 \mathrm{r} / \mathrm{min}$, the culture medium was removed. After centrifugation, the supernatant was discarded, and the precipitate was inoculated into the wastewater and then put into the shaker at $36^{\circ} \mathrm{C}$ and $220 \mathrm{rpm} / \mathrm{min}$, after treatment for $10 \mathrm{~h}$, take out and let stand for $15 \mathrm{~min}$, take $5 \mathrm{ml}$ of supernatant to determine COD, and take samples of the remaining water samples after pretreatment to determine Ammonia nitrogen. The results of this round of screening experiment are shown in Table 2.

Table 2Results of the second round of screening experiment

\begin{tabular}{|c|c|c|c|c|c|c|}
\hline Number & Blank & $(1)$ & $(2)$ & $(3)$ & $(4)$ & $(5)$ \\
\hline $\mathrm{COD}_{\mathrm{Cr}}(\mathrm{mg} / \mathrm{L})$ & 1625.7 & 1249.0 & 1066.1 & 1280.3 & 245.6 & 301.2 \\
\hline $\mathrm{NH}_{4}-\mathrm{N}(\mathrm{mg} / \mathrm{L})$ & 64.2 & 60.5 & 59.4 & 62.1 & 27.5 & 25.8 \\
\hline
\end{tabular}

According to the calculation of the experimental results, compared with the water sample, the COD in the blank group increased by $166.8 \%$, and the ammonia nitrogen decreased by $3.7 \%$ (1) In group A, COD increased by $107.5 \%$ and ammonia nitrogen decreased by $6.0 \%$ (2) In group A, COD increased by $92.7 \%$ and ammonia nitrogen decreased by $7.8 \%$ (3) In group A, COD increased by $111.7 \%$ and ammonia nitrogen decreased by 6.7\% (4) COD and ammonia nitrogen decreased by $59.4 \%$ and $56.7 \%$ respectively (5) COD and ammonia nitrogen decreased by $50.2 \%$ and $60.6 \%$ respectively.

According to the analysis of the calculation results, compared with the blank group, the COD and ammonia nitrogen of the five groups of bacteria decreased, indicating that the five bacteria can grow in the wastewater added with nutrients. Compared with the water samples, COD in the wastewater treated by the five microbial agents increased due to the addition of more glucose. The increase of COD in group (4) and (5) was the least(4) Compared with group (5), the decrease of ammonia nitrogen was the highest. According to the above data analysis, the processing efficiency of (4) and (5) groups was significantly higher than that of the other three groups.

\section{Determinationg of Growth Curve of High Efficiency Bacteria}

\subsection{Determination method}

Select (4) and (5) groups of microbial flora with better degradation effect, take $0.1 \mathrm{ml}$ bacterial solution from the 
prepared bacterial solution, inoculate and sterilize the broth liquid medium, and put it into the shaking table at $36{ }^{\circ} \mathrm{C}$ and 220rpm / min for cultivation. From 0h, take samples every 2 hours, measure the absorbance with UV-vis spectrophotometer at the wavelength of $600 \mathrm{~nm}$, take the uninoculated liquid culture medium as blank, dilute the high concentration bacterial solution with water, keep the absorbance between 0.3 and 0.8 , calculate the OD value of the sample according to formula (3.1) after the absorbance determination, and draw the growth curve according to the data.

$$
\mathrm{OD}=\mathrm{a} \times \mathrm{d}-\mathrm{a}_{0}
$$

Where: a -- the measured value of sample absorbance;

d -- dilution ratio of sample;

$\mathrm{a}_{0^{--}}$Blank absorbance measurement value.

4.2 Results and discussion

After determination, the absorbance of undiluted blank medium is 0.024 , and the determination results of COD removal bacteria (4) group of petrochemical wastewater are shown in Table 3.

Table 3 Results of OD value determination of group bacteria

\begin{tabular}{|c|c|c|c|c|c|c|c|}
\hline Sampling time & 0 & 2 & 4 & 6 & 8 & 10 & 12 \\
\hline Absorbance & 0.037 & 0.037 & 0.041 & 0.207 & 0.538 & 0.728 & 0.674 \\
\hline Dilution ratio & 1 & 1 & 1 & 1 & 1 & 4 & 8 \\
\hline OD & 0.037 & 0.037 & 0.041 & 0.207 & 0.538 & 2.922 & 5.372 \\
\hline Sampling time & 14 & 16 & 18 & 20 & 22 & 24 & \\
\hline Absorbance & 0.882 & 0.555 & 0.469 & 0.535 & 0.587 & 0.649 & \\
\hline Dilution ratio & 8 & 16 & 16 & 16 & 16 & 16 & \\
\hline OD & 7.056 & 4.44 & 7.504 & 8.56 & 9.392 & 10.384 & \\
\hline
\end{tabular}

(4) The growth curve is shown in Figure 1.

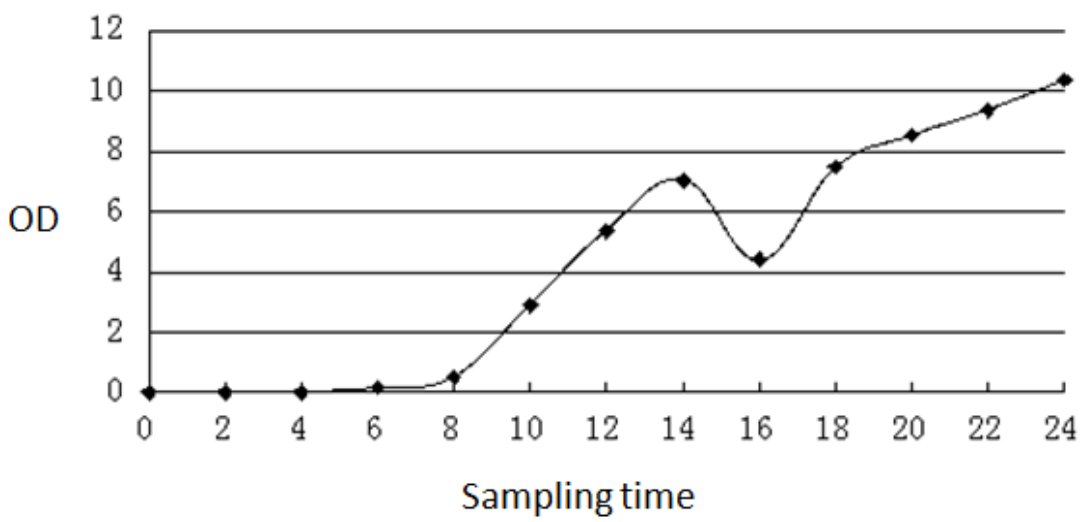

Fig 1: Growth curve of group (4)

From the shape of the growth curve, (4) the microbial agent is a compound microbial agent containing two kinds of non-synchronous growth microorganisms. The first microbial adaptation period is $8 \mathrm{~h}$, reaching the logarithmic period at $8 \mathrm{~h}$, and the logarithmic period lasts to the 14th h. at the end of the logarithmic period, the OD value can reach 7.0, and it enters the decay period at $14 \mathrm{~h}$. The second microorganism could be detected in logarithmic phase at $16-18 \mathrm{~h}$, and the OD value reached 7.5 at the end of logarithmic phase. At $18 \mathrm{~h}$, it entered a stable period, and the decay period of the second microorganism was not detected within $24 \mathrm{~h}$.

The determination results of chemical wastewater COD treatment bacteria (5) are shown in Table 4. 
Table 4 (5) Results of OD value determination of group bacteria

\begin{tabular}{|c|c|c|c|c|c|c|c|}
\hline Sampling time & 0 & 2 & 4 & 6 & 8 & 10 & 12 \\
\hline Absorbance & 0.03 & 0.032 & 0.031 & 0.035 & 0.084 & 0.254 & 0.407 \\
\hline Dilution ratio & 1 & 1 & 1 & 1 & 1 & 4 & 8 \\
\hline OD & 0.006 & 0.008 & 0.007 & 0.011 & 0.06 & 0.992 & 3.232 \\
\hline Sampling time & 14 & 16 & 18 & 20 & 22 & 24 & \\
\hline Absorbance & 0.712 & 0.474 & 0.526 & 0.553 & 0.563 & 0.573 & \\
\hline Dilution ratio & 8 & 16 & 16 & 16 & 16 & 16 & \\
\hline OD & 5.665 & 7.545 & 8.409 & 8.85 & 8.969 & 9.17 & \\
\hline
\end{tabular}

(5) The growth curve is shown in Figure 2

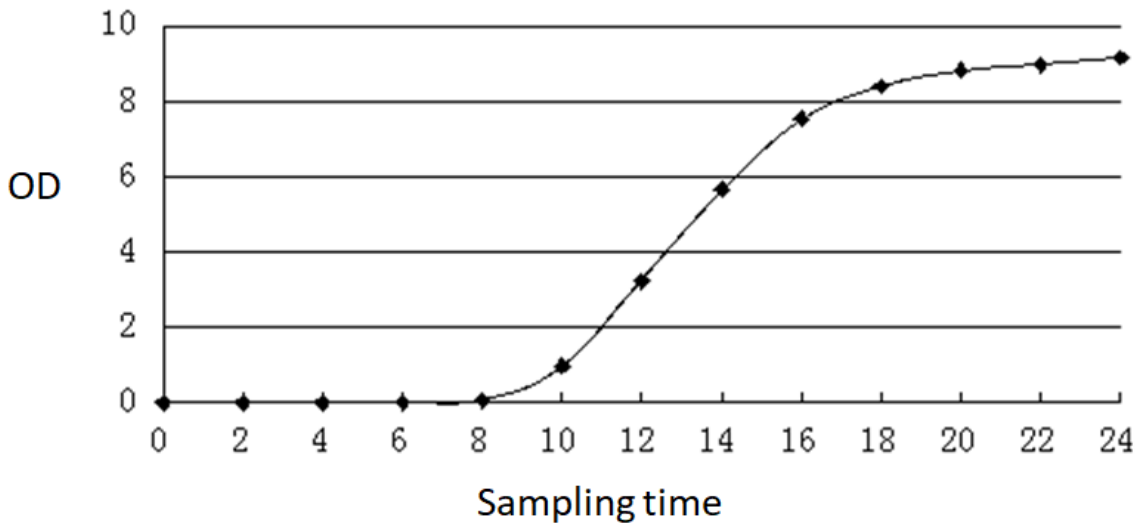

Fig 2:(5) growth curve of $\mathrm{HG}$

According to the growth curve of (5), the agent was a single agent, which entered the logarithmic phase from the 9th hour, and the logarithmic phase lasted for 8 hours. The OD value reached 8.0 at the end of logarithmic period. No death period was detected within 24 hours.

\section{Conclusion}

After the research of this experiment, the following conclusions are obtained

1.After screening experiment, two kinds of bacteria were screened out from five kinds of bacteria, which were (4) Bacillus licheniformis+Pediococcus(5) Bacillus licheniformis + Enterococcus.

2.Through the treatment effect verification experiment, it can be seen that (4) group of bacteria has the best treatment effect on CODCR, the removal rate can reach 59.4\%, and the removal rate of $\mathrm{NH}_{4}-\mathrm{N}$ is $56.7 \%$ (5) $\mathrm{The}$ removal rate of $\mathrm{NH}_{4}-\mathrm{N}$ and CODCR was $50.2 \%$ and $60.6 \%$, respectively. The above removal rate data are the best data monitored in the experiment.

3. Through the growth curve test, it can be seen that (4) group of microbial agents is a compound microbial agent containing two kinds of non-synchronous growth microorganisms. The first microbial adaptation period is $8 \mathrm{~h}$, reaching the logarithmic period at $8 \mathrm{~h}$, and the logarithmic period lasts to the $11 \mathrm{~h}$, and then enters the decline period at $14 \mathrm{~h}$. The second microorganism could be detected in logarithmic phase at $16-18 \mathrm{~h}$, and entered into stationary phase at $18 \mathrm{~h}$. The decay phase of the second microorganism was not detected within 24h(5) Group A was a single bacterial agent, which entered logarithmic phase from $9 \mathrm{~h}$, and the logarithmic phase lasted for $8 \mathrm{~h}$. At the end of the $17 \mathrm{~h}$ logarithmic phase, it entered a stationary phase, and its decay period was not detected within $24 \mathrm{~h}$. 


\section{References}

[1] H.I.Ul, W.Ahmad, I. Ahmad, et al.,"Photocatalytic oxidative degradation of hydrocarbon pollutants in refinery wastewater using TiO2 as catalyst," Water Environment Research, vol. 92, no. 12, 2020.

[2] A.R.L.Yuri, B.M.Priscila, R.M.Victor, et al.,"Osmotic membrane bioreactor (OMBR) in refinery wastewater treatment: The impact of a draw solute with lower diffusivity in the process performance,"Chemical Engineering Journal, vol. 406, 2021.

[3] F.Arshad, M.Selvaraj, F.Banat, et al.,"Removal of metal ions and organics from real refinery wastewater using double- functionalized graphene oxide in alginate beads," Journal of Water Process Engineering, vol. 38, 2020.

[4] K.Yang,J.W. Yu,Q.Y.Guo,et al.,"Treatment per-formance comparison between regular O3-BAC and O3-BAC with rear sand filtration:Verification in a full-scale study,” Environ Sci Eur, vol. 319, no. 1, pp. $1-9,2019$.

[5] J.Zheng, T.Lin, W. Chen, et al.,"Removal of precursors of typical nitrogenous disinfection by products in ozonation integrated with biological activated carbon," Chemosphere,vol. 209, pp. 68 - 77, 2018.

[6] A. Fathima, S. Munirasu, B. Fawzi, et al., "Removal of metal ions and organics from realrefinery wastewater using double- functionalized graphene oxide in alginate beads," Journal of Water Process Engineering, vol. 38, 2020.

[7] S.Ghosh, S.Chakraborty, “Aerobic granulation of single strain oil degraders: Salt tolerance enhancing organics and nitrogen removal from high-strength refinery wastewater," Journal of Water Process Engineering, vol. 42, 2021. 